Check for updates

Cite this: RSC Adv., 2019, 9, 34401

\title{
Prediction of pressure-induced phase transformations in $\mathrm{Mg}_{3} \mathrm{As}_{2} \dagger$
}

\author{
Kang Yang, Jingming Shi, (D) * Shicong Ding, Ruiming Su, Wenwen Cui, Meiling Xu, \\ Jian $\mathrm{HaO}^{*}$ and Yinwei Li
}

Pressure is a fundamental tool that can induce structural and electronic transformations, which is helpful to search for exotic materials not accessible at ambient conditions. Here, we have performed an extensive structural study on cubic $\mathrm{Mg}_{3} \mathrm{As}_{2}$ in a pressure range of $0-100 \mathrm{GPa}$ by using a combination of structure predictions and first-principle calculations. Interestingly, two novel structures with space groups C2/m and $P \overline{1}$ were uncovered that become energetically most stable at pressures of $12 \mathrm{GPa}$ and $30 \mathrm{GPa}$, respectively. Phonon dispersions demonstrate that the three phases are dynamically stable in their respective low-enthalpy pressure ranges. The electronic calculations show that $\mathrm{Mg}_{3} \mathrm{As}_{2}$ keeps semiconductor properties at pressures up to $100 \mathrm{GPa}$. The interesting thing is that the direct semiconducting property of $\mathrm{Mg}_{3} \mathrm{As}_{2}$ transforms into indirect semi-conducting when the pressure is above $12 \mathrm{GPa}$. The current results provide new insights for understanding the behavior of $\mathrm{Mg}_{3} \mathrm{As}_{2}$ at high pressures.

Received 14th August 2019

Accepted 25th September 2019

DOI: $10.1039 / c 9 r a 06341 b$

rsc.li/rsc-advances

phases of $\mathrm{Mg}_{3} \mathrm{P}_{2}$ in the $\mathrm{A}_{3} \mathrm{~B}_{2}$-type family that are stable in the

\section{Introduction}

Pressure is considered as one of the most fundamental thermodynamic variables which can be used to influence the structural configuration, electronic properties and synthesis of unusual stoichiometric materials. ${ }^{1-9}$ These fruitful results indicate that pressure is a powerful tool for discovering and designing novel materials which possess unique properties, like being super conductive, super hard, and transparent semiconductive.

Group II alkaline metal elements typically form $A_{3} B_{2}$-type compounds with the elements in group $\mathrm{V}$. These compounds have attracted much attention due to their wide band gaps and can be viewed as good materials in electronic and optoelectronic devices. $\mathrm{Mg}_{3} \mathrm{~N}_{2}$, a typical $\mathrm{A}_{3} \mathrm{~B}_{2}$-type compound, has been identified to crystallize in the cubic anti-bixbyite structure of the mineral $(\mathrm{Mn}, \mathrm{Fe})_{2} \mathrm{O}_{3}$ (ref. 10) with space group $I a \overline{3}$ at ambient conditions. Experimental studies have shown that the cubic $\mathrm{Mg}_{3} \mathrm{~N}_{2}$ undergoes several phase transformations ${ }^{\mathbf{1 1 , 1 2}}$ to $C 2 / m^{13}$ and $P \overline{3} m 1$ (ref. 13) at high pressure. While for another compound in this group, $\mathrm{Ca}_{3} \mathrm{~N}_{2}$, numerous phases have been synthesized at the experimental level or predicted in theoretical simulations, namely $I a \overline{3},{ }^{14,15} R \overline{3} c,{ }^{16,17} \mathrm{Pbcn},{ }^{18} \mathrm{C} / \mathrm{m}^{19}$ $P \overline{3} m 1,{ }^{19}$ and $I \overline{4} 3 d .^{20}$ In 2017 , a phase transition of $\mathrm{Mg}_{3} \mathrm{P}_{2}$ was found by theoretical simulation when going from ambient conditions to high pressure. ${ }^{21}$ It reveals that there are four

School of Physics and Electronic Engineering, Jiangsu Normal University, Xuzhou, China.E-mail: jingmingshi@jsnu.edu.cn; jian_hao@jsnu.edu.cn

$\dagger$ Electronic supplementary information (ESI) available. See DOI: $10.1039 / \mathrm{c} 9 \mathrm{ra06341b}$ pressure range from the ambient to $100 \mathrm{GPa}$. The previous studies have shown that most of the structures of the $A_{3} B_{2}$-type family possess $I a \overline{3}$ symmetry which is the typical configuration of rare-earth sesquioxides (e.g., $\mathrm{Sc}_{2} \mathrm{O}_{3},{ }^{22} \mathrm{Y}_{2} \mathrm{O}_{3},{ }^{23} \mathrm{Er}_{2} \mathrm{O}_{3},{ }^{24}$ $\mathrm{Gd}_{2} \mathrm{O}_{3},{ }^{25} \mathrm{In}_{2} \mathrm{O}_{3}$ (ref. 26)). As, the sister element to $\mathrm{N}$ and $\mathrm{P}$ elements in group VA, exhibits the similar electron-cloud out of the core and possesses similar chemical properties. In early 1964, Juza and Kroebel experimentally found two phases of $\mathrm{Mg}_{3} \mathrm{As}_{2}$, namely $I a \overline{3}$ and $P \overline{3} m 1,{ }^{27}$ which were also reported by Ali Mokhtari and Matin Sedighi in 2010 with simulation ${ }^{28}$ and collected by the Materials Project database. ${ }^{29}$ However, the As atom has a much larger ionic radius than $\mathrm{N}$ and $\mathrm{P}$ atoms, which may affect the structural configuration of arsenide and the phase transformation sequence, especially at high pressure. On the other hand, the existent compounds of the $\mathrm{A}_{3} \mathrm{~B}_{2}$ type family usually have large band gaps, which is beneficial to potential applications. For example, the band gap of $\mathrm{Mg}_{3} \mathrm{~N}_{2}$ (ref. 13) is about $1.65 \mathrm{eV}$ at ambient conditions and $2.35 \mathrm{eV}$ at about $25 \mathrm{GPa}$, respectively. While for the $\mathrm{Mg}_{3} \mathrm{P}_{2}$ compound, ${ }^{21}$ the gap is about $1.73 \mathrm{eV}$ and $1.14 \mathrm{eV}$ at ambient conditions and $2.5 \mathrm{GPa}$, respectively. When the pressure is above $35 \mathrm{GPa}$, $\mathrm{Mg}_{3} \mathrm{P}_{2}$ transforms into a metallic phase. Thus, there is a lot of necessity for us to study the band gap evolution and the potential applications of $\mathrm{Mg}_{3} \mathrm{As}_{2}$ from ambient conditions to high pressure. Finally, as an antistructure in comparison to the traditional $\mathrm{A}_{2} \mathrm{~B}_{3}$-type, accurate structural configuration and the phase transition sequence of $\mathrm{Mg}_{3} \mathrm{As}_{2}$ under high pressure can provide important implications for understanding the compressional behavior of related materials like rare-earth sesquioxides. 
In order to clarify the phase transitions and potential electronic applications of $\mathrm{Mg}_{3} \mathrm{As}_{2}$ compounds from ambient conditions to high pressure, we performed systematic structural searches by using a structural prediction method combining with the first-principles method. We predicted two novel structures of $\mathrm{Mg}_{3} \mathrm{As}_{2}$ that are unknown in the pressure range from ambient to $100 \mathrm{GPa}$. The phonon dispersions show that all the novel phases of $\mathrm{Mg}_{3} \mathrm{As}_{2}$ were dynamically stable. Electronic property calculations show that all the phases of $\mathrm{Mg}_{3} \mathrm{As}_{2}$ can be viewed as semiconductors. Interestingly, with the pressure increasing, the character of direct semiconductor (for $P \overline{3} m 1$ and $C 2 / m$ phases) will turn into an indirect band gap property $(P \overline{1}$ phase).

\section{Methods}

For the structure stability, we used two steps to get the ground state structure at each pressure. Firstly, as the elements with similar electron clouds always form the same structure configuration, we chose the known phases of $\mathrm{Mg}-\mathrm{N}$ and $\mathrm{Mg}-\mathrm{P}$ (like $I a \overline{3}, C 2 / m$ and $P \overline{3} m 1$ ) as prototype structures to predict the novel phase of the $\mathrm{Mg}$-As system. Secondly, we performed structure predictions to search all the possible structures in the energy surface. For the structure prediction we used the CALYPSO ${ }^{30,31}$ method with the cells containing 2 to 4 formula units in a pressure range from 0 to $100 \mathrm{GPa}$. The underlying $a b$ initio structural relaxations and the electronic band structure calculations were performed in the framework of density functional theory (DFT) using the VASP code. ${ }^{32}$ The calculations were carried out in the generalized gradient approximation ${ }^{33}$ for the exchange-correlation potential, using the Perdew-Burke-Ernzerhof $^{34}$ (PBE) functional. The electronic wave functions were expanded in a plane wave basis set with a cutoff energy of $600 \mathrm{eV}$. The electron-ion interaction was described by means of projector augmented wave (PAW) ${ }^{35}$ pseudopotentials with $2 \mathrm{p}^{6} 3 \mathrm{~s}^{2}$ and $3 \mathrm{~s}^{2} 3 \mathrm{p}^{3}$ electrons in the valence band for the $\mathrm{Mg}$ and As atoms, respectively. Monkhorst-Pack $k$-point ${ }^{36}$ meshes with a grid density of $0.03 \AA^{-1}$ were chosen to achieve a total energy convergence of better than $1 \mathrm{meV}$ per atom. The phonon dispersion curves were computed by the direct supercell calculation method, as implemented in the PHONOPY program. $^{37}$

\section{Results and discussions}

To investigate the phase transitions of $\mathrm{Mg}_{3} \mathrm{As}_{2}$, we performed structural prediction with unit-cell sizes of maximal four formula units (f.u.) at pressures of $0,20,50$ and $100 \mathrm{GPa}$, respectively. Due to computational restrictions, we decided to use a suitable unit-cell size to ensure the calculation efficiency. For the next step, we chose at least 5 structures in each prediction for further accurate geometric optimization. The phase transitions of $\mathrm{Mg}_{3} \mathrm{As}_{2}$ under high pressure are further illustrated in Fig. 1, where we plot the relative enthalpies per formula unit with respect to the $C 2 / \mathrm{m}$ phase as a function of pressure, including the enthalpies of metastable phases that we predicted. From Fig. 1, we can clearly find that the $I a \overline{3}$ phase is

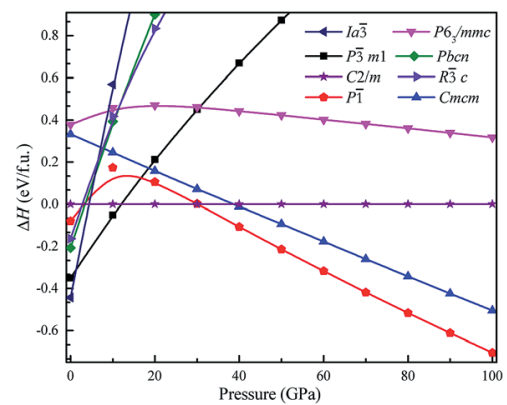

Fig. 1 Relative enthalpy per formula unit of the different phases of $\mathrm{Mg}_{3} \mathrm{As}_{2}$ with respect to the $\mathrm{C} 2 / \mathrm{m}$ phase as a function of pressure.

the ground-state for $\mathrm{Mg}_{3} \mathrm{As}_{2}$ at ambient conditions. The crystal structure of this phase is shown in Fig. 2(a). The $I a \overline{3}$ phase has an interesting configuration with $\mathrm{Mg}$-As bond lengths of 2.62$2.73 \AA$ A. There are eight formula units in its primitive cell and all the $\mathrm{Mg}$ atoms are in the same Wyckoff positions (48e) while two different As atoms possess the (8b) and (24d) positions. In this phase, each $\mathrm{Mg}$ atom connects with four As atoms, while each As atom is bonded to six $\mathrm{Mg}$ atoms. However, the Ia $\overline{3}$ phase is quickly destabilized by pressure, and a novel structure with space group $P \overline{3} m 1$ becomes the ground-state at a pressure of 1.3 GPa. The crystal configuration of this phase is shown in Fig. 2(b). This phenomenon also emerges in the similar system of $\mathrm{Mg}_{3} \mathrm{P}_{2} \cdot{ }^{21}$ The $P \overline{3} m 1$ phase has five atoms in its primitive cell. The $\mathrm{Mg}$ atoms possess Wyckoff positions of (2d) and (1a), while the As atoms only locate at (2d) positions. The $\mathrm{Mg}$ atoms and As atoms form an octahedron and tetrahedron with $\mathrm{Mg}-\mathrm{As}$ bond lengths of 2.62-2.73 $\AA$. These behaviors are in good agreement with the information that was presented in the Materials Project

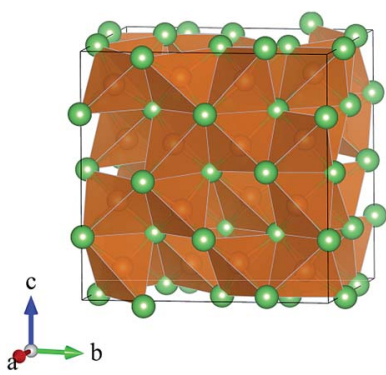

(a)
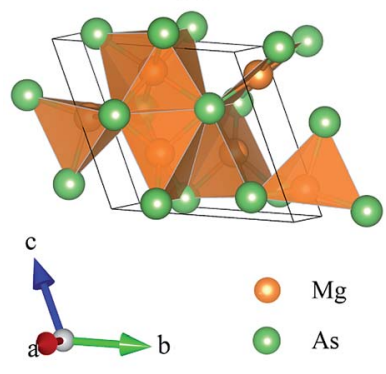

(c)

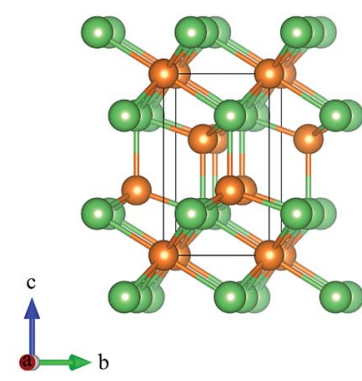

(b)

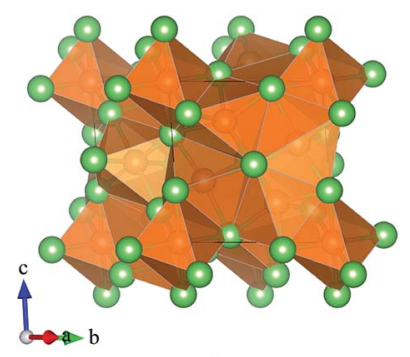

(d)
Fig. 2 Crystal structures of $\mathrm{Mg}_{3} \mathrm{As}_{2}$ (orange: $\mathrm{Mg}$ and green: $\mathrm{As}$ ); (a) the la3 phase at ambient pressure, (b) the $P 3 \mathrm{~m} 1$ phase at $5 \mathrm{GPa}$, (c) the $\mathrm{C} 2 /$ $m$ phase at $15 \mathrm{GPa}$ and (d) the $P 1$ phase at $30 \mathrm{GPa}$. 
database. ${ }^{29}$ When the pressure increases, the $C 2 / m$ phase becomes energetically favorable at around $12 \mathrm{GPa}$. The $C 2 / \mathrm{m}$ structure has two formula units in its unit cell, as shown in Fig. 2(c). This is a remarkable structure which is composed of a regular octahedron and a tetrahedron. They connect to each other with a sharing edge formed by two As atoms. The distances between the $\mathrm{Mg}$ atom and As atom are 2.47-2.73 $\AA$ at $20 \mathrm{GPa}$. With the pressure increasing to $30 \mathrm{GPa}$, we predict a novel phase with $P \overline{1}$ symmetry which is energetically favored in a large range of pressures from about $30 \mathrm{GPa}$ to more than $100 \mathrm{GPa}$, the maximum pressure we considered. The $P \overline{1}$ phase has an interesting structural configuration with several octahedrons and they connect with each other by sharing one or two faces, as shown in Fig. 2(d). The distances between the Mg and As atoms are around 2.50-2.65 $\AA$ under $30 \mathrm{GPa}$. Thus, we propose a clear phase transformation sequence of $\mathrm{Mg}_{3} \mathrm{As}_{2}$ from ambient conditions to high pressure. The sequence is as follows: $\quad I a \overline{3} \stackrel{1.3 \mathrm{GPa}}{\longrightarrow} P \overline{3} m 1 \stackrel{12 \mathrm{GPa}}{\longrightarrow} C 2 / m \stackrel{30 \mathrm{GPa}}{\longrightarrow} P \overline{1}$. Comparing with the series of phase transitions of $\mathrm{Mg}_{3} \mathrm{~N}_{2}$ $(I a \overline{3} \stackrel{20.6 \mathrm{GPa}}{\stackrel{2.5 \mathrm{GPa}}{\longrightarrow}} C 2 / m \underset{35 \mathrm{GPa}}{\stackrel{67 \mathrm{GPa}}{\longrightarrow}} P \overline{3} m 1)^{13} \underset{65 \mathrm{GPa}}{\text { and }} \mathrm{Mg}_{3} \mathrm{P}_{2}$ $\left(\mathrm{Ia} \overline{3} \stackrel{2.5 \mathrm{GPa}}{\longrightarrow} P \overline{3} m 1 \stackrel{35 \mathrm{GPa}}{\longrightarrow} P 6_{3} / m m c \stackrel{65 \mathrm{GPa}}{\longrightarrow} C 2 / c\right),{ }^{21}$ we can find that these three compounds adopt the same ambient structure, while high pressure phases vary accordingly, which is caused by the different ionic radii of $\mathrm{N}, \mathrm{P}$ and As atoms. The lattice parameters and atomic coordinates which are optimized for the most energetically favorable structures are listed in Table 1. The lattice parameters of $I a \overline{3}(a=12.16 \AA)$ are in good agreement with the data in the Materials Project $(a=12.176$ $\AA),{ }^{29}$ which indicates that our calculations are credible. To examine the interactions between $\mathrm{Mg}$ and As atoms of the new phases, we calculated the Bader charges, as shown in Table 1. The results show that all the phases of $\mathrm{Mg}_{3} \mathrm{As}_{2}$ compound exhibit ionic behavior and each $\mathrm{Mg}$ atom will lose about 1.50e while each As atom will gain about 2.25e. To understand the bonding behaviors of these structures, we calculated the electron localization function (ELF) and the charge density difference, as shown in the ESI. $\dagger$ The results show that the charges almost localize around the As atoms that form anions, while $\mathrm{Mg}$ atoms act as cations. This is also in good agreement with the results of the Bader analysis.

We also calculated the lattice parameters and volumes of these four novel phases of $\mathrm{Mg}_{3} \mathrm{As}_{2}$ as a function of pressure, as shown in Fig. 3. It reveals that all the lattice constants will decrease when the pressure increases and detects that the volume collapses at the phase transitions: $I a \overline{3} \rightarrow P \overline{3} m 1$ (at 1.3 $\mathrm{GPa}$ ), $P \overline{3} m 1 \rightarrow C 2 / m$ (at $12 \mathrm{GPa}$ ) and $C 2 / m \rightarrow P \overline{1}$ (at $30 \mathrm{GPa}$ ). This indicates that the three phase transitions in $\mathrm{Mg}_{3} \mathrm{As}_{2}$ are first-order in nature. This phenomenon is in good agreement with the previous works on $\mathrm{Mg}_{3} \mathrm{P}_{2} \cdot{ }^{21}$

In order to ensure the thermal stability of these novel structures, we calculated the phonon dispersions of $I a \overline{3}, P \overline{3} m 1$, $C 2 / m$ and $P \overline{1}$ at $0,1.5,20$ and $30 \mathrm{GPa}$, respectively, as shown in Fig. 4. As expected, they do not exhibit any imaginary phonon frequencies indicating that all the novel phases are mechanically stable. It is well known that the PBE function systematically underestimates band gaps. Therefore we decided to adopt the hybrid exchange-correlation functional of Heyd, Scuseria, and Ernzerhof (HSE06) ${ }^{38}$ to obtain reliable band gaps. The calculated electronic band structures (HSE06) and the projected densities of state (HSE06) are shown in Fig. 5. For comparison,

Table 1 Predicted crystal structures of $\mathrm{Mg}_{3} \mathrm{As}_{2}$ in its $l a \overline{3}, P \overline{3} \mathrm{~m} 1, C 2 / m$ and $P \overline{1}$ phases at selected pressures. Bader charges of these phases at specific conditions are shown in the last column

\begin{tabular}{|c|c|c|c|c|}
\hline Pressure (GPa) & Space group & Lattice parameters & Atomic coordinates (fractional) & Bader charge $(e)$ \\
\hline \multirow[t]{3}{*}{0} & \multirow[t]{3}{*}{$I a \overline{3}$} & $a=b=c=12.46 \AA$ & $\mathrm{Mg}(48 \mathrm{e})(-0.11,-0.36,0.88)$ & 1.51 \\
\hline & & $\alpha=\beta=\gamma=90^{\circ}$ & As $(8 b)(-0.25,-0.25,0.75)$ & -2.26 \\
\hline & & & As $(24 \mathrm{~d})(-0.02,-1.00,0.25)$ & -2.26 \\
\hline \multirow[t]{3}{*}{5} & \multirow[t]{3}{*}{$P \overline{3} m 1$} & $a=b=4.19 \AA$ & $\mathrm{Mg}(2 \mathrm{~d})(0.33,0.67,0.36)$ & 1.50 \\
\hline & & $c=6.55 \AA$ & $\operatorname{Mg}(1 \mathrm{a})(0.00,0.00,0.00)$ & 1.56 \\
\hline & & $\alpha=\beta=90^{\circ}, \gamma=120^{\circ}$ & As $(2 d)(0.33,0.67,0.77)$ & -2.28 \\
\hline \multirow[t]{6}{*}{15} & \multirow[t]{6}{*}{$C 2 / m$} & $a=13.84 \AA$ & $\mathrm{Mg}(4 \mathrm{i})(0.34,0.50,0.02)$ & 1.49 \\
\hline & & $b=3.89 \AA$ & $\mathrm{Mg}(4 \mathrm{i})(0.25,0.00,0.66)$ & 1.52 \\
\hline & & $c=7.15 \AA$ & $\operatorname{Mg}(2 \mathrm{c})(0.50,0.50,0.50)$ & 1.55 \\
\hline & & $\alpha=116.67^{\circ}$ & $\mathrm{Mg}(2 \mathrm{~b})(0.50,0.00,1.00)$ & 1.53 \\
\hline & & $\beta=105.80^{\circ}$ & As $(4 \mathrm{i})(0.13,0.50,0.74)$ & -2.29 \\
\hline & & $\gamma=90^{\circ}$ & As $(4 \mathrm{i})(0.40,0.50,0.73)$ & -2.26 \\
\hline \multirow[t]{11}{*}{30} & \multirow[t]{11}{*}{$P \overline{1}$} & $a=6.77 \AA$ & $\mathrm{Mg}(2 \mathrm{i})(0.52,0.53,0.72)$ & 1.56 \\
\hline & & $b=6.80 \AA$ & $\mathrm{Mg}(2 \mathrm{i})(0.23,0.23,0.15)$ & 1.56 \\
\hline & & $c=7.14 \AA$ & $\mathrm{Mg}(2 \mathrm{i})(0.09,0.77,0.61)$ & 1.54 \\
\hline & & $\alpha=93.32^{\circ}$ & $\mathrm{Mg}(2 \mathrm{i})(0.97,0.67,0.25)$ & 1.55 \\
\hline & & $\beta=97.04^{\circ}$ & $\mathrm{Mg}(2 \mathrm{i})(0.50,0.79,0.110)$ & 1.55 \\
\hline & & $\gamma=65.85^{\circ}$ & $\mathrm{Mg}(1 \mathrm{a})(0.00,0.00,0.00)$ & 1.54 \\
\hline & & & $\mathrm{Mg}(1 \mathrm{f})(0.50,0.00,0.50)$ & 1.58 \\
\hline & & & As $(2 \mathrm{i})(0.33,0.95,0.80)$ & -2.35 \\
\hline & & & As (2i) $(0.21,0.89,0.32)$ & -2.31 \\
\hline & & & As (2i) $(0.79,0.43,0.04)$ & -2.37 \\
\hline & & & As (2i) $(0.75,0.61,0.51)$ & -2.26 \\
\hline
\end{tabular}




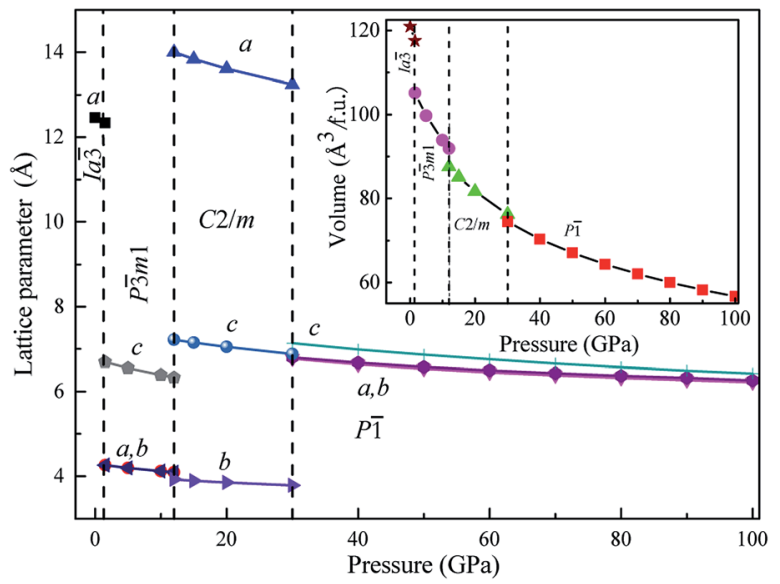

Fig. 3 Lattice parameters and volume of $\mathrm{Mg}_{3} \mathrm{As}_{2}$ as a function of pressure.

the calculated band structures of all structures by using the PBE function are shown in the ESI. $\uparrow$ From our calculation, it is evident that all the four phases of $\mathrm{Mg}_{3} \mathrm{As}_{2}$ are semiconductors and the band gap will decrease with increasing pressure. For example, at $0 \mathrm{GPa}$, the band gap of the $I a \overline{3}$ phase is $1.42 \mathrm{eV}$ (PBE) and $2.08 \mathrm{eV}$ (HSE06), which is in good agreement with the previous work, ${ }^{28}$ while it will become $0.86 \mathrm{eV}$ (PBE) and $1.43 \mathrm{eV}$ (HSE06) for $P \overline{3} m 1$ at $1.5 \mathrm{GPa}, 0.68 \mathrm{eV}$ (PBE) and $1.21 \mathrm{eV}$ (HSE06) for $C 2 / m$ at $20 \mathrm{GPa}$ and $0.49 \mathrm{eV}$ (PBE) and $0.93 \mathrm{eV}$ (HSE06) for $P \overline{1}$ at $30 \mathrm{GPa}$, respectively. Comparing to another two compounds $\left(\mathrm{Mg}_{3} \mathrm{~N}_{2}\right.$ (ref. 13) and $\mathrm{Mg}_{3} \mathrm{P}_{2}$ (ref. 21)), $\mathrm{Mg}_{3} \mathrm{As}_{2}$ has a larger band gap at ambient conditions and can remain a semiconductor even at high pressure. These good performances can make $\mathrm{Mg}_{3} \mathrm{As}_{2}$ compounds act as good potential semiconductors at both ambient conditions and high pressure. The projected densities of state show that the electrons near the Fermi level are almost all contributed by the As atoms, which reveals that the As atoms play an important role in the semiconductor property.

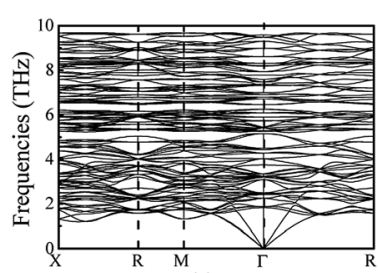

(a)

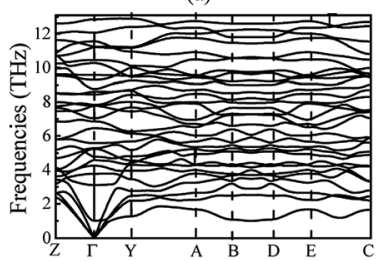

(c)

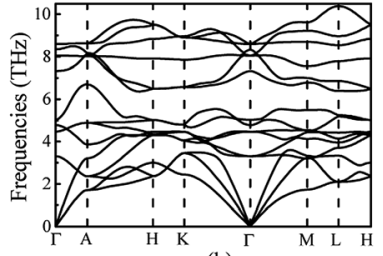

(b)

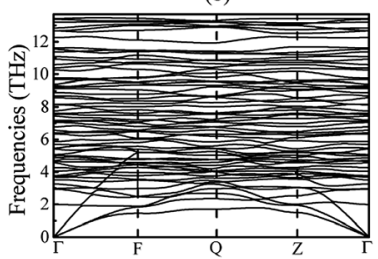

(d)
Fig. 4 Phonon dispersions of $\mathrm{Mg}_{3} \mathrm{As}_{2}$ for (a) the la3 phase at ambient pressure, (b) the $P 3 \mathrm{~m} 1$ phase at $1.5 \mathrm{GPa}$, (c) the $C 2 / \mathrm{m}$ phase at $20 \mathrm{GPa}$ and (d) the $P 1$ phase at $30 \mathrm{GPa}$.
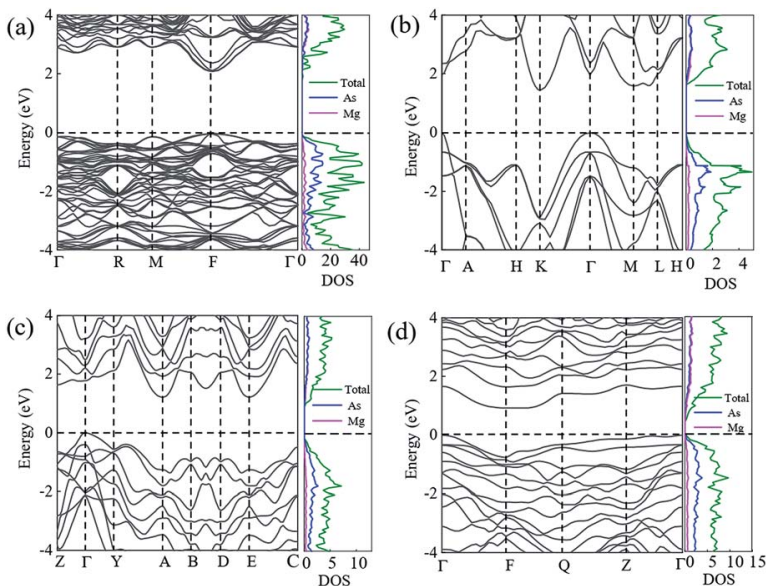

Fig. 5 Electronic band structures and densities of state (HSE06) of $\mathrm{Mg}_{3} \mathrm{As}_{2}$ for (a) the la $\overline{3}$ phase at ambient pressure, (b) the $P \overline{3} \mathrm{~m} 1$ phase at $1.5 \mathrm{GPa}$, (c) the $C 2 / \mathrm{m}$ phase at $20 \mathrm{GPa}$ and (d) the $P \overline{1}$ phase at $30 \mathrm{GPa}$.

\section{Conclusions}

In summary, using a global structural prediction method we studied systematically the phase transitions and electronic properties of $\mathrm{Mg}_{3} \mathrm{As}_{2}$. We identified two novel phases with unexpected structures that might be experimentally synthesizable over a wide range of pressures. The novel phases have $C 2 / m$ and $P \overline{1}$ symmetry under high pressure. The transition pressures of the two structures are $12 \mathrm{GPa}$ and $30 \mathrm{GPa}$, respectively. The Bader charge calculations show that all the phases possess ionic behavior. The phonon dispersions reveal that they are mechanically stable. From the calculations of electronic properties we can indicate that all the phases are semiconductor. These results show remarkable phase transitions of $\mathrm{Mg}_{3} \mathrm{As}_{2}$, and can serve as an important guide for further experimental studies of this compound.

\section{Conflicts of interest}

There are no conflicts to declare.

\section{Acknowledgements}

J. S. and W. C. acknowledge the Project Funded by the National Natural Science Foundation of China under Grant No. 11804129 and No. 11804128 and the Jiangsu Normal University under Grant No. 18XLRS003 and No. 18XLRS004. Y. L. and J. H. acknowledge funding from the National Natural Science Foundation of China under Grant No. 11722433 and No. 11404148 and Qing Lan Project of Jiangsu Province. All the calculations were performed using the High Performance Computing Center of the School of Physics and Electronic Engineering of Jiangsu Normal University. The crystal structures were visualized with VESTA. ${ }^{39}$

\section{References}

1 L. Zhang, Y. Wang, J. Lv and Y. Ma, Nat. Rev. Mater., 2017, 2, 17005. 
2 Y. Ma, M. Eremets, A. R. Oganov, Y. Xie, I. Trojan, S. Medvedev, A. O. Lyakhov, M. Valle and V. Prakapenka, Nature, 2009, 458, 182-185.

3 Y. Li, Y. Wang, C. J. Pickard, R. J. Needs, Y. Wang and Y. Ma, Phys. Rev. Lett., 2015, 114, 125501.

4 Y. Li, J. Hao, H. Liu, Y. Li and Y. Ma, J. Chem. Phys., 2014, 140, 899.

5 A. Drozdov, M. Eremets, I. Troyan, V. Ksenofontov and S. Shylin, Nature, 2015, 525, 73.

6 D. Duan, Y. Liu, F. Tian, D. Li, X. Huang, Z. Zhao, H. Yu, B. Liu, W. Tian and T. Cui, Sci. Rep., 2014, 4, 6968.

7 Y. Li, X. Feng, H. Liu, J. Hao, S. A. Redfern, W. Lei, D. Liu and Y. Ma, Nat. Commun., 2018, 9, 722.

8 Y. Li, J. Hao, H. Liu, S. Lu and J. S. Tse, Phys. Rev. Lett., 2015, 115, 105502.

9 J. Shi, W. Cui, S. Botti and M. A. L. Marques, Phys. Rev. Mater., 2018, 2, 023604.

10 M. Stackelberg and R. Paulus, Z. Phys. Chem. B, 1933, 22, 305-322.

11 D. Partin, D. Williams and M. O'Keeffe, J. Solid State Chem., 1997, 132, 56-59.

12 F. Zong, C. Meng, Z. Guo, F. Ji, H. Xiao, X. Zhang, J. Ma and H. Ma, J. Alloys Compd., 2010, 508, 172-176.

13 J. Hao, Y. Li, Q. Zhou, D. Liu, M. Li, F. Li, W. Lei, X. Chen, Y. Ma, Q. Cui, et al., Inorg. Chem., 2009, 48, 9737-9741.

14 H. Moissan, Compt. Rend., 1898, 127, 497-501.

15 O. Reckeweg and F. J. DiSalvo, Z. Anorg. Allg. Chem., 2001, 627, 371-377.

16 H. H. Franck, M. A. Bredig and G. Hoffmann, Naturwissenschaften, 1933, 21, 330-331.

17 H. Hartmann and H. J. Fröhlich, Z. Anorg. Allg. Chem., 1934, 218, 190-192.

18 R. S. Bradley, D. C. Munro and M. Whitfield, J. Inorg. Nucl. Chem., 1966, 28, 1803-1812.

19 J. Hao, Y. Li, J. Wang, C. Ma, L. Huang, R. Liu, Q. Cui, G. Zou, J. Liu and X. Li, J. Phys. Chem. C, 2010, 114, 16750-16755.

20 C. Braun, S. L. Börger, T. D. Boyko, G. Miehe, H. Ehrenberg, P. Höhn, A. Moewes and W. Schnick, J. Am. Chem. Soc., 2011, 133, 4307.
21 B. Liu, J. Hao, X. Tang and Y. Li, J. Alloys Compd., 2017, 720, 207-211.

22 T. Schleid and G. Meyer, J. Less-Common Met., 1989, 149, 7380.

23 B. Antic, P. Oennerud, D. Rodic and R. Tellgren, Powder Diffr., 1993, 8, 216-220.

24 Q. Guo, Y. Zhao, C. Jiang, W. L. Mao, Z. Wang, J. Zhang and Y. Wang, Inorg. Chem., 2007, 46, 6164-6169.

25 F. Zhang, M. Lang, J. Wang, U. Becker and R. Ewing, Phys. Rev. B: Condens. Matter Mater. Phys., 2008, 78, 064114.

26 H. Yusa, T. Tsuchiya, J. Tsuchiya, N. Sata and Y. Ohishi, Phys. Rev. B: Condens. Matter Mater. Phys., 2008, 78, 092107.

27 R. Juza and R. Kroebel, Z. Anorg. Allg. Chem., 1964, 331, 187199.

28 A. Mokhtari and M. Sedighi, Phys. Rev. B: Condens. Matter Mater. Phys., 2010, 405, 1715-1720.

29 A. Jain, S. P. Ong, G. Hautier, W. Chen, W. D. Richards, S. Dacek, S. Cholia, D. Gunter, D. Skinner, G. Ceder, et al., APL Mater., 2013, 1, 011002.

30 Y. Wang, J. Lv, L. Zhu and Y. Ma, Phys. Rev. B: Condens. Matter Mater. Phys., 2010, 82, 094116.

31 Y. Wang, J. Lv, L. Zhu and Y. Ma, Comput. Phys. Commun., 2012, 183, 2063-2070.

32 G. Kresse and J. Hafner, Phys. Rev. B: Condens. Matter Mater. Phys., 1993, 47, 558.

33 G. Kresse and D. Joubert, Phys. Rev. B: Condens. Matter Mater. Phys., 1999, 59, 1758.

34 J. P. Perdew, K. Burke and M. Ernzerhof, Phys. Rev. Lett., 1996, 77, 3865.

35 G. Kresse and D. Joubert, Phys. Rev. B: Condens. Matter Mater. Phys., 1999, 59, 1758.

36 H. J. Monkhorst and J. D. Pack, Phys. Rev. B: Solid State, 1976, 13, 5188.

37 A. Togo, F. Oba and I. Tanaka, Phys. Rev. B: Condens. Matter Mater. Phys., 2008, 78, 134106.

38 J. Heyd, G. E. Scuseria and M. Ernzerhof, J. Chem. Phys., 2003, 118, 8207-8215.

39 K. Momma and F. Izumi, J. Appl. Crystallogr., 2011, 44, 12721276. 